\title{
In silico study of whey-acidic-protein domain containing oral protease inhibitors
}

\author{
YOSHIKO IDOJI $^{1}$, YUKO WATANABE ${ }^{1,2}$, AKIFUMI YAMASHITA ${ }^{2}$, KYOSUKE YAMANISHI $^{3}$, \\ SEIJI NISHIGUCHI ${ }^{1}$, KAZUNORI SHIMADA ${ }^{1,3}$, TERUO YASUNAGA ${ }^{2}$ and HIROMICHI YAMANISHI ${ }^{1}$ \\ ${ }^{1}$ Hirakata Ryoikuen, Tsudahigashi 2-1-1, Hirakata, Osaka 573-0122; ${ }^{2}$ Department of Genome Research, \\ Genome Information Research Center, Research Institute for Microbial Diseases, Osaka University, Suita, \\ Osaka 565-0871; ${ }^{3}$ Laboratory of Developmental Biology and Reproduction, Institute for Advanced Medical \\ Sciences, Hyogo College of Medicine, Mukogawa 1-1, Nishinomiya, Hyogo 663-8501, Japan
}

Received November 19, 2007; Accepted December 21, 2007

\begin{abstract}
Since whey-acidic-protein domain (WAP) containing protease inhibitors such as SLPI (secretory leukocyte protease inhibitor) and elafin (elastase-specific inhibitor) have antimicrobial activities and are thought to play critical roles in mucosal defenses, we are interested in these protease inhibitors. By accessing the Novartis mouse expression database, we found that the four WAP family members, SLPI, WFDC2, WFDC5, and WFDC12, are highly expressed in the oral organs, such as the trachea, tongue, and salivary glands. Since their WAP domains play pivotal roles in the antimicrobial and/or antiprotease activities and their application in therapeutics are expected to have practical value, we collected $98 \mathrm{WAP}$ homologues and tried to predict their physiological functions by analyzing their amino acid sequence structures. From the multiple alignments of amino acid sequences, we predicted that most of the mammalian $\mathrm{N}$-terminal WAP domains derived from SLPIs and the WAP domains derived from WFDC12s have antimicrobial activities, whereas most of the mammalian C-terminal WAP domains derived from SLPIs and the WAP domains derived from elafins have antiprotease activities. From the phylogenetic tree, it was revealed that an ancestral WAP protein initially diverged into the WFDC5-C WAP domain and the ancestral protein for the other WAP domains. Subsequently, the ancestral protein
\end{abstract}

Correspondence to: Dr Hiromichi Yamanishi, Hirakata Ryoikuen, Tsudahigashi 2-1-1, Hirakata, Osaka 573-0122, Japan

E-mail: hirochan@hirakataryoiku-med.or.jp

Abbreviations: elafin, elastase-specific inhibitor; SLPI, secretory leukocyte protease inhibitor; WAP, whey-acidic-protein; WFDC, WAP four-disulphide core; -C or -N, C-terminal WAP or N-terminal WAP

Key words: antimicrobial activity, antiprotease activity, elafin, SLPI, whey-acidic-protein, C-terminal WAP, N-terminal WAP, WFDC for the other WAP domains diverged into two ancestral proteins, one for elafin and SLPI-C WAP domains and the other, for SLPI-N, WFDC15b, WFDC12, and WFDC5-N WAP domains, respectively. Moreover, the tree indicated that the WFDC5-N and WFDC12 WAP domains share a common ancestral protein.

\section{Introduction}

Protease inhibitors such as SLPI (secretory leukocyte protease inhibitor) and elafin (elastase-specific inhibitor) are synthesized and secreted by local tissues and/or organs and are known to have antimicrobial activities (1). These two protease inhibitors contain the whey-acidic-protein domain (WAP) and these domains are known to play pivotal roles in the antimicrobial and/or antiprotease activities (1). Since SLPI is thought to limit the spread of fungi, bacteria, and viruses on mucocutaneous surfaces in vivo by blocking colonization of transient pathogens and/or their release of destructive proteases such as elastase in oral sites (2), we are interested in the protease inhibitors within the oral cavity with an aim of future therapeutic applications.

We hypothesized that genes highly expressed in the tongue, salivary glands, snout epidermis, and trachea contribute to maintenance of the levels of salivary protease inhibitors. Since the expression levels of 36,182 mRNAs have been estimated for 61 different mouse organs, tissues, and/or cells by two independent experiments, and the results are stocked as a dataset in the Mouse GNF1M, we initiated our study by accessing the Novartis expression database (3), and found that four WAP family members, SLPI, WFDC2, WFDC5, and WFDC12, are highly expressed in oral organs. Subsequently, we collected 98 WAP homologues and tried to predict their physiological functions by analyzing their amino acid sequence structures. We aligned their amino acid sequences, and tried to elucidate the relationship between the amino acid sequences and their functions. We also constructed a phylogenetic tree and revealed an initial divergence of WFDC5-C-terminal WAP from an ancestral WAP protein. Based on these analyses, we discuss the relationships between the amino acid sequences and antiprotease and/or antimicrobial activities. 
Table I. Classification of clones highly expressed in the oral organs.

\begin{tabular}{lcccc}
\hline $\begin{array}{l}\text { Oral organ } \\
\text { (abbreviation) }\end{array}$ & $\begin{array}{c}\text { Organ- } \\
\text { specific clones }\end{array}$ & $\begin{array}{c}\text { Not functionally } \\
\text { annotated clones }\end{array}$ & $\begin{array}{c}\text { Protease inhibitor } \\
\text { clones }\end{array}$ & $\begin{array}{c}\text { WAP family } \\
\text { members }\end{array}$ \\
\hline Salivary gland (Sg) & 774 & $214(27.6 \%)$ & $4(0.5 \%)$ & WFDC12 \\
Snout epidermis (Se) & 277 & $28(10.1 \%)$ & $5(1.8 \%)$ & WFDC2 \\
Trachea (Tr) & 186 & $24(12.9 \%)$ & $5(1.6 \%)$ & SLPI and WFDC5 \\
Tongue (To) & 183 & $24(13.1 \%)$ & $4(3.1 \%)$ & $5(20.0 \%)$ \\
To and Se & 131 & $6(4.6 \%)$ & 0 & 0 \\
To and Tr & 25 & 0 & 0 & 0 \\
Sg and Se & 5 & 0 & 0 & $4(0.2 \%)$ \\
Sg and Tr & 1 & 0 & $26(1.6 \%)$ & \\
Tr and Se & 1 & 0 & 0 & \\
Tr, Se and To & 20 & $296(18.5 \%)$ & & \\
\hline Totals & 1603 & & & \\
\end{tabular}

Not functionally annotated clones include unclassifiable clones, clones coding for hypothetical proteins, and clones corresponding to DNA segments. Protease inhibitor clones include 14 serpin family members, 4 WAP family members, 2 cystatins, and 6 different protease inhibitors.

\section{Materials and methods}

Selection of oral organ-specific cDNA clones. Oral organspecific cDNA clones were selected from the expression database 'Mouse GNF1M', which contains gene expression data for 36,182 mouse genes obtained from two independent experiments using 61 organs, tissues, and/or cells (http:// wombat.gnf.org/index.html) (3). Log-transformed ratio data for the 61 organs, tissues, and/or cells were normalized. The clone was denoted as 'oral organ-specific' if the normalized ratio value exceeded +2 SD for the microarray data of the tongue, salivary gland, snout epidermis, and trachea in both of the two independent experiments $(3,5)$.

Selection of cDNA clones coding for WAP domain containing protease inhibitors. After annotating the oral organ-specific cDNA clones, we looked for clones coding for protease inhibitors using the names of mouse protease inhibitor genes described as key words in the review of Puente and LopezOtin (6), and selected clones coding for WAP domaincontaining protease inhibitors using WFDC, WAP, elafin, and/or SLPI as key words.

Nomenclature. The nomenclature of primate WFDC-encoding proteins and genes are written in uppercase letters as described in Clauss et al (4). The corresponding proteins and genes from other vertebrates or mammals are also written with uppercase letters and numbers in this study.

Collecting the homologues of the $N$ - and C-terminal WAP domains of mouse SLPI. We performed PSI-BLAST homology searches using either the mouse SLPI N-WAP domain with 45 amino acid residues or its C-WAP domain with 47 amino acid residues as queries against the database at the NCBI (7). After running PSI-BLAST for three iterations, we collected those clones showing an Expert (E)-value $<0.001$ as N-terminal
WAP homologues and those showing an E-value $<0.004$ as C-terminal WAP homologues. The lower the E-value, or the closer it is to zero, the more 'significant' the match is (7). The WAP domains were collected as follows: the NCBI conserved domain database (CDD version 2.1.1) (8) was downloaded and conserved domains in each WAP homologue were identified using the reverse position-specific BLAST (rpsBLAST) program (v1.65) (7). Identified domains were then extracted using the perl script named extractCD. The web version of the extractCD is available from: http://www.gen-info.osaka-u.ac.jp/ uhmin/study/extractCD/ index.html

Multiple alignments and prediction of functions carried by WAP homologues. Multiple alignments were performed with CLUSTAL W (9). Functions carried by each of the WAP homologues were predicted by comparing amino acid sequences of the WAP homologues (test sequences) to WAP domains with experimentally demonstrated antimicrobial or antiprotease activities (control sequences). After these sequences were aligned with CLUSTAL W, the levels of homology were scored by analyzing the aligned control and test sequences using the newly developed CCResid program. The scores were used to arrange the aligned sequences. The web version of the CCResid program is available from: http://www .gen-info.osaka-u.ac.jp/ uhmin/study/ccresid/ index.html

Phylogenetic tree. Phylogenetic and molecular evolutionary analyses were conducted by the neighbor-joining (NJ) method (10) in MEGA version 4.0 (11) or CLUSTAL W (9).

\section{Results and Discussion}

Isolation of cDNA clones highly expressed in the oral organs and selection of clones encoding WAP domain-containing 
Table II. The WAP family proteins studied.

\begin{tabular}{|c|c|c|c|c|c|}
\hline \multirow[b]{2}{*}{ Scientific name } & \multirow[b]{2}{*}{ Abbreviation } & \multicolumn{4}{|c|}{ Clone ID (gi numbers) } \\
\hline & & SLPI & WFDC5 & WFDC12 & Elafin \\
\hline Aotus nancymaae & NMonkey & 134093146 & 134093142 & 134093143 & 134093144 \\
\hline Bos taurus & Cattle & & 119906045 & 3132274 & \\
\hline Callithrix jacchus & Marmoset & 134093120 & 134093115 & 134093116 & 134093117 \\
\hline Canis familiaris & Dog & & 73992498 & & 57104238 \\
\hline Chlorocebus aethiops & VMonkey & 134093163 & 134093158 & 134093159 & 134093160 \\
\hline Colobus guereza & CMonkey & 134093077 & & 134093073 & 134093074 \\
\hline Gorilla gorilla gorilla & Gorilla & 134093094 & 134093089 & 134093090 & 134093091 \\
\hline Homo sapiens & Human & 4507065 & 21717822 & 119596281 & 4505787 \\
\hline Lemur catta & Lemur & 134093102 & 134093098 & 134093099 & 134093100 \\
\hline Macaca mulatta & RMonkey & 109091820 & 109091843 & 134093108 & 109091835 \\
\hline Monodelphis domestica & Opossum & & 126303379 & & \\
\hline Mus musculus & Mouse & 6755574 & 18043977 & 20149778 & \\
\hline Ornithorhynchus anatinus & Duckbill & & 149637518 & & \\
\hline Otolemur garnettii & Galago & 134093085 & 134093082 & 134093083 & \\
\hline Ovis aries & Sheep & 78126182 & 37528750 & & \\
\hline Pan troglodytes & Chimpanzee & 134093069 & & & \\
\hline Papio anubis & OBaboon & 134093053 & 134093048 & 134093049 & 134093050 \\
\hline Phacochoerus aethiopicus & Warthog & & & & 4887638 \\
\hline Pongo pygmaeus & Orangutan & 134093132 & 134093128 & 134093129 & 134093130 \\
\hline Rattus norvegicus & Rat & 5802680 & 27704652 & 57114316 & \\
\hline Saimiri boliviensis boliviensis & BSMonkey & 134093155 & 134093150 & 134093151 & 134093152 \\
\hline Sus scrofa & Pig & & & & 2501659 \\
\hline
\end{tabular}

$\mathrm{N}$ - and C-terminal WAP domains were extracted from each SLPI and WFDC5 sequence, whereas only one WAP domain was extracted from each WFDC12 and elafin sequence (see Materials and methods). There was one exception, the N-terminal WAP domain was not extracted from Colobus guereza SLPI (gil134093077). The WAP domain extracted from mouse WFDC15b (gil38174311) was used as one of the antimicrobial activity positive controls (see Fig. 1A), and the WAP domains extracted from chicken (gil118100817) and nematode (gil72000192) were used as out group proteins for the phylogenetic tree construction (Fig. 3).

protease inhibitors. We selected cDNA clones highly expressed in the salivary gland, tongue, trachea, and snout epidermis, and isolated 1,603 clones from 36,182 clones. We found that $296(18.5 \%)$ of the 1,603 clones had not been functionally annotated (Table I), and determined that $26(1.6 \%)$ of the clones encode protease inhibitors as described by Puente and Lopez-Otin (6): four of the 26 coded for WAP domain containing proteins, i.e., SLPI, WFDC2, WFDC5, and WFDC12 (4) (Table I). Since we are interested in WAP domain-containing salivary protease inhibitors with antimicrobial and/or antiprotease activities, we focused our study on these four WAP motif-containing proteins. All four of these genes are mapped on mouse chromosome 2 and share significant homology (4).

Structures and functions of WAP domains derived from SLPIs and WFDCs. To obtain insights into the relation between antimicrobial and/or antiprotease activities and the amino acid residues of the WAP domains, we collected 98 WAP homologues (Table II): 70 were derived from primates (12), 11 from rodents, 8 from ungulata, 3 from dog, 2 each from opossum and duckbill, and one each from chicken and nematode. These isolates included WAP homologues derived from SLPIs, WFDC5s, WFDC12s, and elafins (Table II). However, no WAP homologues derived from WFDC2 were included, indicating that the homologies between WAPs derived from WFDC2s and those derived from SLPIs are significantly lower. Although we were not able to isolate a cDNA clone corresponding to the mouse elafin gene from screening the Novartis mouse database, we isolated many WAP homologues derived from elafins of primates, carnivora, and ungulata in our homology search (Table II). This result is consistent with the study that most surprisingly, there is no mouse elafin gene (4).

Antimicrobial activity (Fig. 1A and B). To elucidate the relationship between the amino acid residues and protein functions, we aligned the amino acid sequences of the human SLPI-N-, mouse WFDC12-, and WFDC15b-WAP domains, because these three WAP domains have been demonstrated to have antimicrobial activities $(1,13)$ (Fig. 1A). This alignment revealed that eight Cys residues as well as Pro11, Pro24, 
(A) Positive sequences

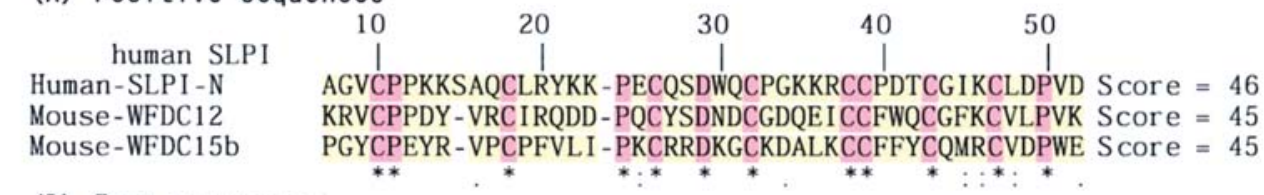

(B) Test sequences

Gorilla-SLPI-N AGVCPPKKSAQCLRYKK-PECQSDWQCPGKKRCCPDTCGIKCLDPVD Score $=46$ Ch i mpanzee-SLPI-N AGVCPPKKSAQCLRYKK-PECQSDWQCPGKKRCCPDTCGIKCLDPVD Score $=46$ Orangutan-SLPI-N AGVCPPKKSAQCLRYEK-PECQSDWQCLGKKRCCPDTCGIKCLDPVD Scor e $=44$ VMonkey-SLPI-N AGVCPPKKSAQCLRYEK-PECQSDWQCLGKKRCCPDICGIKCLDPVD Score $=43$ OBaboon-SLPI-N AGVCPPKKSAQCLRYEK-PECQSDWQCLGKKRCCPDICGIKCLDPVD Score $=43$ RMonkey-SLPI - N AGVCPPKKSAQCLRYEK-PQCQSDWQCLGKKRCCPDICGIKCLDPVD Score $=43$ NMonkey-SLPI -N AGVCPPRKSAQCLRYEK-PECQSDWQCPEKKRCCPDICGIKCLDPVD Score $=42$ BSMonkey-SLPI -N AGVCPPRKSAQCLRFEK-AECQSDWQCPEKKRCCPDVCGIKCLDPVD Score $=40$ Marmoset-SLPI - N AGVCPSTKPALCLRYEK-PECQSDWQCPEKKRCCPGICGIKCLDPVD Score $=38$ Sheep-SLPI -N AGACPPRKSAOCFGNEK-PRCSSDWOCPHKKKCCLDTCGTECLDPVN Score $=33^{*}$ Galago-SLPI -N AGACPPRRLAKCPKFEK-PECRSDWQCPEKKRCCTDACSV̄KCLDPID Scor e $=33$ Lemur-SLPI-N AGVCPPKVPAPCLKKEE-RECISDWTCPGSKRCCPHFCGMRCLNPH- Score $=31^{* *}$ Mouse-SLPI-N IGACPAKKPAOCLKLEK-P̄OCRTDWECPGKORCCODACGSKCVNPVP Score $=31 * *$ Rat -SLPI - N IGACPARKPAQCLKLEK-PECGTDWECPGKQRCCQDTCGFKCLNPVP Score $=31$

Rat - WFDC 12

BSMonkey-WFDC 12 OBaboon-WFDC12 NMonkey-WFDC12 Marmoset -WFDC12 Orangutan-WFDC12 VMonkey-WFDC12 Gorilla-WFDC12 Human-WFDC12 CMonkey-WFDC12 RMonkey-WFDC12 Lemur-WFDC12

Duckbi 11 -WFDC5-N Opossum- WFDC5 - N NMonkey-WFDC5 - N Gal ago-WFDC5-N Lemur-WFDC5-N Human-WFDC5 - N Gor illa-WFDC5-N BSMonkey-WFDC5-N Marmoset - WFDC5-N Rat - WFDC5-N Cat t le-WFDC5-N Dog-WFDC5-N Mouse-WFDC5-N VMonkey-WFDC5 -N OBaboon-WFDC5-N RMonkey-WFDC5 - N
KGVCPPDN-VRCIRGED-PQCHNDNDCKDQKICCYWHCGFKCVQPV- Score $=36$ PGVCPADN-VRCIKSDP-PQCHTDQDCQGIRKCCYLHCGFKCVIPV- Score $=29$ AGVCPADN-VRCFKSDP-PQCHTDQDCLGARKCCYLHCGFKCVIPV-Score $=29$ PEVCPADN-VRCIKSDP-PQCHTDODCOGIRKCCYLHCGFKCVIPV- Score $=28$ PGVCPADN-IRCIKSDP-PQCHTDQDCQGIRKCCYLHCGFKCVIPV- Score $=28$ AGVCPADN-VRCFKSDP-PQCHTDQDCLGERKCCYLHCGFKCVIPV- Score $=28$ AGVCPADN -VRCFKSDP-PQCHTDQDCLGERKCCYLHCGFKCVIPV- Score $=28$ AGVCPADN-VRCFKSDP-PQCHTDQDCLGERKCCYLHCGFKCVIPV- Score $=28$ AGVCPADN-VRCFKSDP-PQCHTDQDCLGERKCCYLHCGFKCVIPV- Score $=28$ AGVCPADN-VRCFKSNP-PQCHTDQDCLGERKCCYLHCGFKCVIPV- Score $=27$ AGVCPADN - I RCFKSDP-PQCHTDQDCLGERKCCYLHCGFKCVIPV- Score $=27$ WGNCPAEK-GSCIKSGP-SQCHADNDCPGDKKCCFLSCSFKCVSPD- Score $=25$

LGGCPADD-TPCLWQRP-DQCSEDSQCPRLKKCCSRACYRQCLPPVR Score $=22^{* *}$ SGGCPPDD-QVCRPG IP-HQCLVDKQCPKGKKCCQQACFLQCVA--- Score $=21^{* *}$ PGACPPDD-GPCLLSVP-DQCMEDRQCPLTRKCCYRACFRQCVPR- - Score $=20 * *$ PGGCPPDD-GPCLLSVP-D̄QCTDDSQCPSTMKCCPRACFRQR IPR- - Score $=20^{* *}$

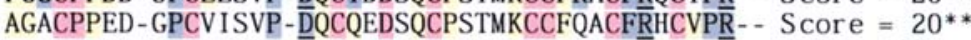
SGGCPPDD-GPCLLSVP-DQCVEDSQCPLTRKCCYRACFRQCVPR- - Score $=19 * *$ SGGCPPDD-GPCLLSVP-DQDCVEDSQCPLTRKCCYRACFRQCVPR- - Score $=19^{* *}$

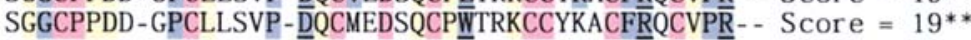
SGRCPPDD-GPCLLSVP-DQCMEDSQCPFFTRKCCYRACFRQCVPR- - Score $=19 * *$ LGGCPPDD-GPCSQVIP-D̄QCANDKQCPSTSKCCSRACFLCCMPR- - Score $=19^{* *}$ SGGCPPDD-GPCLLSVP-DQDCLHDSQCPSGMKCCRQGCFLQCVR-- - S core $=19^{* *}$ FGGCPPDD-GPCLLSVP-DHCMDDSQCPARMKCCSKACFRQCVPM- - Score $=18^{* *}$ LGGCPPDD-GLCNQNTPPD̄QCLNDKQCPSSWKCCRRACFLQCMPR - - Score $=18^{* *}$ WGGCPADD-GPCLLSVP-DQDVEDSQCPLTRKCCYRACFRQCVPR-- Score $=18^{* *}$ WGGCPADD-GPCLLSVP-DQCVEDSQCPLTRKCCYRACFRQCVPR-- Score $=18^{* *}$ WGGCPADD-GPCLLSVP- $\underline{D} Q C V E D S Q C P \underline{L} T R K C C Y R A C F \underline{R} Q C I P \underline{R}--$ Score $=17^{* *}$

Figure 1. Multiple sequence alignment of WAP homologues to the N-terminal WAP domain of mouse SLPI. (A) Positive sequences show alignments of WAP domains demonstrated to have antimicrobial activities. (B) Test sequences show alignments of SLPI-N-WAPs, WFDC12-WAPs, and WFDC5-WAPs. Multiple alignments were performed as described in Materials and methods. For the conservation line output in the CLUSTAL format alignment file, three characters are used: '*' indicates positions that have a single, fully conserved residue; ':' and '.' indicate positions that contain substitutions with highly and with weakly similar amino acid residues, respectively. Red boxes indicate fully conserved residues; yellow boxes, amino acids present within the positive sequences; non-colored boxes, amino acids substituted with highly or weakly similar amino acid residues; grey boxes, amino acids substituted with unrelated amino acid residues. The alignments were arranged according to the arbitrarily defined scores: each red or yellow box for an amino acid is scored as 1 and each grey or non-colored box is scored as zero. The scores marked with ** indicate that the WAPs are predicted not to have similar antimicrobial activities. The underlined amino acid residues correspond to the conserved amino acid residues substituted with unrelated amino acid residues. Names, origins, and abbreviations related to the WAP domains are summarized in Table II. Amino acid residue numbers of human SLPI are shown in panel (A).

Asp29, and Pro50 were completely conserved and that Ala16, Glu25, Gly34, Ile45, Lys46, Leu48, and Asp52 of human SLPI were all replaced by similar amino acid residues among these three WAP domains. Most of these conserved amino acid residues should have pivotal roles in the antimicrobial activities.

Subsequently, we aligned the amino acid sequences of 14 SLPI-N-, 12 WFDC12-, and 16 WFDC5-N-WAP domains (Fig. 1B). The results obtained with 14 SLPI-N- and 12 WFDC12-WAP domains revealed that they maintained nearly all of the above-described conserved amino acid residues (Fig. 1A and B). There were three exceptions: i) Pro24 of Human-SLPI-N was replaced by Arg24 in Lemur-SLPI-N; ii) Ile45 of Human-SLPI-N was replaced by Thr45 in SheepSLPI-N, and iii) by Ser45 in Mouse-SLPI-N (see Fig. 1B, scores marked with $* *)$. These results suggest that the remaining 23 of 26 SLPI-N- and WFDC12-WAP domains retain antimicrobial activities.

On the other hand, although the eight $\mathrm{Cys}$ residues were conserved among the 16 WFDC5-N, several other above- 
(A) Positive sequences

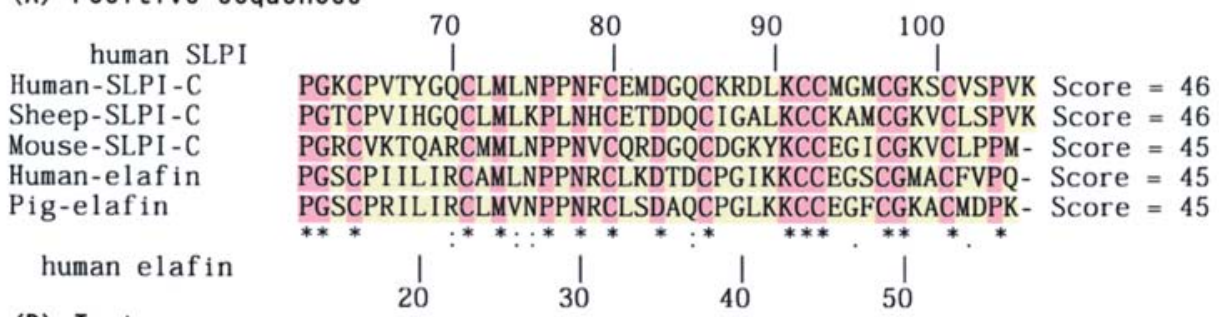

(B) Test sequences

Ch impanzee-SLPI -C PGKCPVTYGQCLMLNPPNFCEMDGQCKRDLKCCMGMCGKSCVSPVK Score $=46$ Gorilla-SLPI-C PGKCPVAYGQCLMLNPPNFCEMDGQCKRNLKCCMGMCGKSCVSPVK Score $=44$ Orangutan-SLPI-C PGKCPVAYGQCLMLNPPNYCEMDGQCERDLKCCMGMCGKSCVSPVK Score $=43$ RMonkey-SLPI-C PGRCPPAYGQCMMLKPPNYCEMDGQCERDLKCCMGMCGKSCVSPVK Score $=42$ OBaboon-SLPI-C PGRCPPAYGQCMMLKPPNYCEMDGQCERDLKCCMGMCGKSCVSPVK Score $=42$ VMonkey-SLPI-C PGRCPPAYGOCMMLNPPNYCEMDGOCERDLKCCMGMCGKSCVSPVK Score $=42$ CMonkey-SLPI-C PGRCPLAYGQCMMLNPPNYCEMDGQCERDLKCCVGMCGKSCVSPVK Score $=41$ Rat -SLPI - C PGRCVKFQGKCLMLNPPNKCQNDGQCDGKYKCCEGMCGKVCLPPV- Score $=41$ BSMonkey-SLPI -C AGKCPAVYGOCMMINPPNHCESDGOCERDLKCCMGMCGKTCVSPL- Score $=38$ Galago-SLPI-C PGACPVVHGRCMM INPPNHCERDGQCQDDFKCCMGMCGKACVSPT- Score $=38$ NMonkey-SLPI-C SGKCPVVYGQCMM IDPPNHCELDGQCERDLKCCVGMCGKTCVSPL- Score $=36$ Marmoset-SLPI-C PGKCPVVYGQCMMIDPPNHCESDGQCEHDMKCC IGMCGKTCVSPL- Score $=36$ Lemur - SLPI - C PGTCPAVYGYCMMLNPSNSCESDGQCQAKFKCCVGMCGKICSSPVE Score $=34^{* *}$

Gorilla-elafin OBaboon-elafin RMonkey-elaf in CMonkey-elaf in VMonkey-elaf in NMonkey-elaf in BSMonkey-elaf in Orangutan-elafin Marmoset-elaf in Galago-elaf in War thog-elaf in Sheep-elaf in Lemur-elaf in Dog-elaf in Cat t le-Trappin-6

NMonkey-WFDC5-C Mouse-WFDC5-C OBaboon-WFDC5-C RMonkey-WFDC5-C VMonkey-WFDC5-C BSMonkey-WFDC5-C Marmoset - WFDC5-C Orangutan-WFDC5-C Human-WFDC5-C Rat-WFDC5-C Gal ago-WFDC5-C Lemur -WFDC5-C Duckbi11-WFDC5-C Gorilla-WFDC5-C Dog-WFDC5-C Cat t le-WFDC5-C Opossum-WFDC5-C
PGSCPVILIRCAMLNPPNRCLKDTDCPGIKKCCEGSCGMACFVPO- Score $=45$ PGSCPNILIRCAMLNPPNRCLKDTDCPGIKKCCEGSCGMACMVPO- Score $=44$ PGSCPNILIRCAMLNPPNRCLKDTDCPGI IKCCEGSCGMACMVPQ- Score $=44$ PGSCPNILIRCAMLNPPNRCLKDTDCPGI IKCCEGSCGMACMVPQ- Score $=44$ PGSCPNILIRCAMLNPPNHCLKDTDCPGIKKCCEGSCGMACMVPO- Score $=44$ SGSCPKTL IRCAMLNPPNRCLKDTDCPG I KKCCEGSCGMACLDPQ- Score $=44$ SGSCPKILIQCAMLNPPNRCLKDTDCPG IKKCCEGSCGMACLDPQ- Score $=44$ PGFCPNILIRCAMLNPPNRCLKDTDCPGIKKCCEGSCGMACFVPO- Score $=43$ SGSCPKTL IRCAMLNPPNRCLKDTDCPG I KKCCEGSCGMACLDPR- Score $=43$ SGSCPNILIRCAMLNPPNHCLSDTQCPGTKKCCEGSCGKRCMDPQ- Score $=41$ PGSCPRILIRCMMVNPPNRCLSDAQCPGVKKCCEGFCGKECLNPR- Score $=41$ RGSCPRVLIRCAMMNPPNRCLRDAQCPGAKKCCEGSCGKTCMDPQ- Score $=41^{* *}$ $\bar{P} G S C P N I L I R C A M L N P P N R C G S D G E C P G S K K C C E G S C G R A C L N P Q-$ Score $=39$ PGSCPPILIQCAMLNPPNRCLSDTECPGARKCCKGPCGLACLQPQ- SCOre $=39$ RGLCPRVRIHCNLWNPPNQCWRDAHCPGAKKCCEGFCGKTCMNPR- SCOre $=31 * *$

LGSCPEDQLRCLSPMN -HLCHKDADCSGKKRCCSSACGRDCRDPVR SCore $=25^{* *}$ SGKCPVDQLRCLSPTK-HMCNKDSDCSGKKRCCASACGRDCRDPS- Score $=24^{* *}$ PGSCPQDQLRCLSPMN̄ - HLCHKDSDCSGKKRCCHSACGRDCR्DPAR Score $=24^{* *}$ PGSCPQDQLRCLSPMN - HLCHKDSDCSGKKRCCHSACGRDCRDDPAR Score $=24^{* *}$ PGSCPQDQLRCLSPVN - HOCHKDSDCSGKKRCCHSACGRDCRDPAR SCOre $=24 * *$ LGSCPEDQLRCLSPMN-HLCHKDADCSGKKRCCPSACGRDCR̄DPAR Score $=24^{* *}$ LGSCPEDQLQCLSPMN - HLCHKDADCSGKKRCCPSACGRDCĒDPAR Score $=24^{* *}$ LGSCPEDQLRCLSPMN-HLCHKDSDCSGKKRCCHSACGRDCRDDPAR Score $=23^{* *}$ LGSCPEDQLRCLSPMN - HLCHKDSDCSGKKRCCHSACGRDCR्RPPAR Score $=23^{* *}$ LGKCPVDQLHCLSPRK-HLCDKDLDCSGKKRCCVSACGRDCRDDPS- Score $=23^{* *}$ LGSCPVDQLHCLSPTK-HLCHQDSNCSGKKRCCPTACGRDCRDDPVK Score $=23^{* *}$ RGGCPEDQLQCLSPTE-HLCNKDSDCSGKKRCCHTSCGRECRDPAR Score $=23^{* *}$ LGDCPKDDLLCLSPIQ - HLCGKDTDCSGIQKCCLAACGRDCREPAK Score $=23^{* *}$ LGSCPEDQL $\overline{H C L S P M N}-$ HLCHKDSDCSGKKRCCHSACGRDCRDDPAR Score $=22 * *$ PGSCPEDRLQCLSPVQ - HLCHKDADCRGSSRCCLGACGRDCR NPI - Score $=22^{* *}$ TGRCPEDRLRCVSPVQ-HLCSKDSDCQGRKRCCPGACGRDCRNNPV- Score $=21^{* *}$ SGQCPPDPLRCLSATQ - HLCTQDKNCSGLKRCCPTACGRDCR्RPVR SCore $=20^{* *}$

Figure 2. Multiple sequence alignment of WAP homologues to the C-terminal WAP domain of mouse SLPI. (A) Positive sequences show alignments of WAP domains demonstrated to have antiprotease activities. (B) Test sequences show SLPI-C-WAPs, elafin-WAPs, and WFDC5-C-WAPs. The other procedures, abbreviations, and symbols are as described in Fig. 1 and/or in Table II. Amino acid residue numbers of human SLPI and those of human elafin are shown in panel (A)

described conserved amino acid residues were replaced by unrelated residues. For example, out of the 16 WFDC5-NWAPs, Pro24 of Human-SLPI-N was replaced by Asp24 in all 16 WAPs, Ile45 of Human-SLPI-N was replaced by Arg45 in 12 WAPs, and Pro50 of Human-SLPI-N was replaced by Arg50 or Met50 in 14 WAPs (Fig. 1A and B). These results suggest that although the 16 WFDC5-N-WAP domains have conserved tertiary structures, they do not have similar antimicrobial activities, or have antimicrobial activities against different microbes.
Antiprotease activity (Fig. 2A and B). Grutter et al (14) found that human SLPI contacts proteases through Leu72 and Met73 of the 'primary' binding loop, which consists of eight residues from Thr67 to Leu74 (Fig. 2A, SLPI). Tsunemi et al (15) described the primary binding site of human elafin, the $\mathrm{V}$ region, to consist of Ala24, Met25, and Leu26, which correspond to Leu72, Met73, and Leu74 of human SLPI (Fig. 2A, elafin).

Since the human SLPI-C-WAP and WAP domains derived from human and pig elafins have antiprotease activities, we 


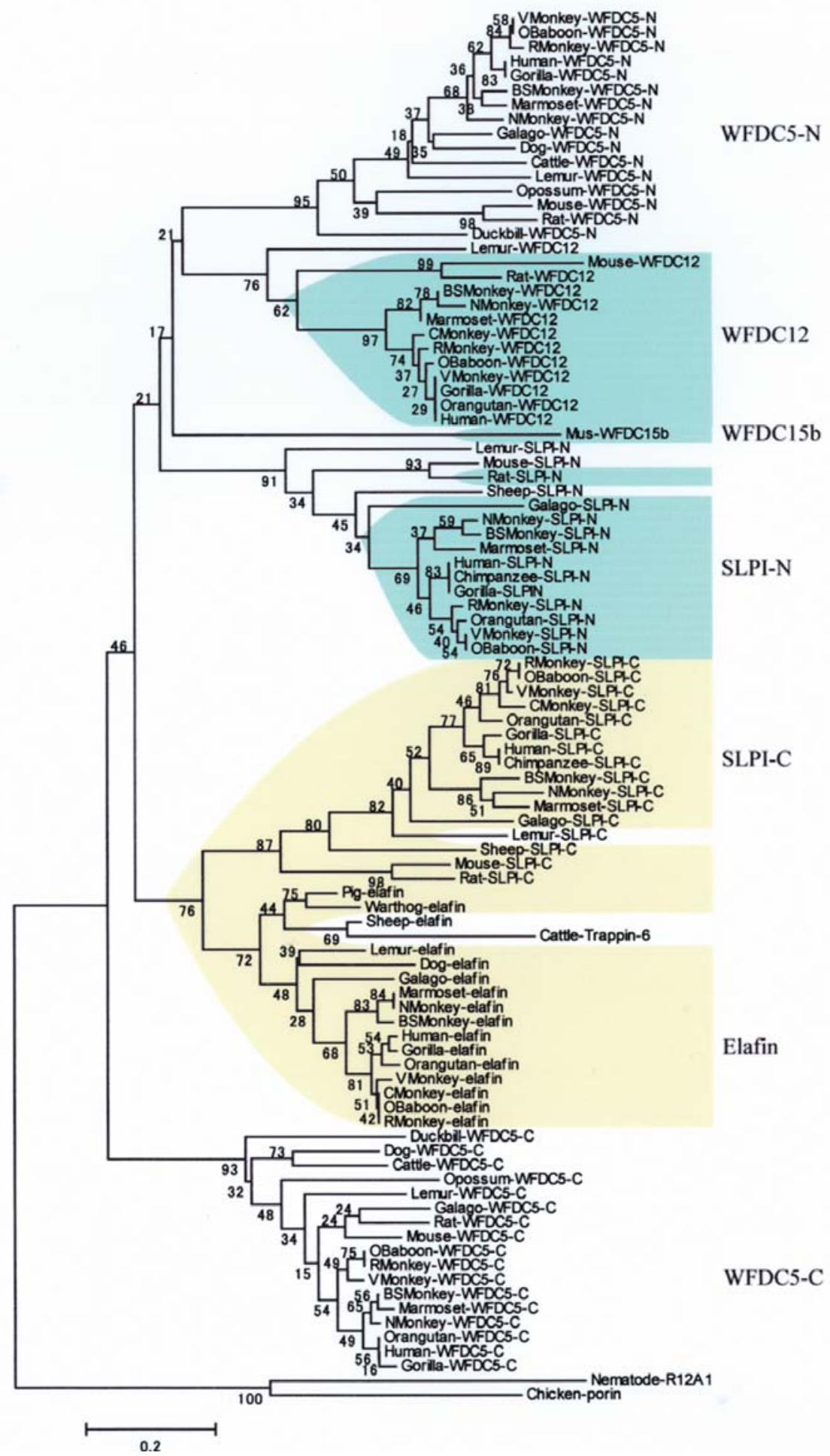

Figure 3. A phylogenetic tree of WAP domains. This tree was constructed by aligning the amino acid sequences of the WAP homologues. Phylogenetic and molecular evolutionary analyses were conducted using the MEGA4 program as described in Materials and methods. Bootstrap values obtained from 1,000 replicates are shown at the corresponding branches by the 1/10 values. The scale bar represents an estimate of the number of amino acid residue substitutions per site. Green colored WAPs are predicted to have antimicrobial activities and yellow-colored WAPs are predicted to have antiprotease activities (see Figs. 1 and 2). The other abbreviations are as described in Table II.

aligned the amino acid sequences of these three WAP domains $(1,16)$ (Fig. 2A). We added the C-WAP domains derived from mouse and sheep SLPIs to this alignment because these two SLPIs have also been demonstrated to have similar antiprotease activities $(17,18)$. The alignment revealed that eight Cys residues as well as Pro61, Gly62, Met73, Pro76, Asn78, Asp83, Lys91, Gly98, and Pro104 were all conserved, and that Gln70, Leu74, Asn75, Gln85, Gly94, and Val102 of human SLPI were all replaced by similar amino acid residues in the other 4 sequences. All these conserved amino acid residues should have pivotal roles in the antiprotease activity (Fig. 2A).

We collected 13 SLPI-C, 15 elafin, and 17 WFDC5-C WAP domains, and aligned their amino acid sequences 
(Fig. 2B). The results obtained with the 13 SLPI-C and 15 elafin WAP domains revealed that they retained almost all of the above-described conserved amino acid residues (Fig. 2A and B). There were three exceptions: i) Gln70 and Val102 of Human-SLPI-C were replaced by Tyr70 and Ser102, respectively, in the Lemur-SLPI-C WAP domain; ii) Pro61 of human elafin was replaced by Arg61 in Sheep-elafin, and iii) Pro61 and Leu74 of Human-SLPI-C were replaced by Arg61 and Trp74, respectively, in Cattle-Trappin-6. Accordingly, we predict that Lemur-SLPI-C-, Sheep-elafin, and Cattle-Trappin-6 WAP domains do not retain antiprotease activities (see Fig. 2, scores marked with **). Although Pro61 of Human-SLPI-C was replaced by Ala61 in BSMonkey-SLPI-C, by Ser61 in Nmonkey-SLPI-C, and by Ser61 in Nmonkey-, BSMonkey-, Marmoset-, and Galagoelafins, these are similar amino acid residues, and these results suggest that these proteins keep their antiprotease activities.

On the other hand, although the eight Cys residues were completely conserved among the 17 WFDC5-C, the amino acid residues of the regions corresponding to the 'primary' binding site of human SLPI (Leu72, Met73, and Leu74 region) and/or to the $\mathrm{V}$ region of human elafin (Ala24, Met25, and Leu26 region) were replaced by different amino acid residues (Fig. 2B) $(13,14)$. These results suggest that the 17 WFDC5-C-WAP domains either have antiprotease activities against different proteases, or do not have antiprotease activities.

Evolution of the WAP domains. To elucidate the evolutionary relationship among WAP domains, we constructed a phylogenetic tree using the amino acid sequences of the WAP domains shown in Figs. 1 and 2 (Fig. 3). Our analysis revealed that an ancestral WAP protein diverged into the WFDC5-CWAP domain and the ancestral protein for the other WAP domains. Brown et al (16) showed a close relationship between elafin-WAP and SLPI-C-WAP domains, and suggested a divergence in their evolution from an ancestral protein subsequent to the divergence of SLPI-N- and SLPI-C-WAP domains. Our results not only support this concept, but also suggest a divergence of WFDC15b-, WFDC12-, and WFDC5N-WAP domains from a diverged ancestral SLPI-N-WAP domain (Fig. 3). Moreover, our results suggest that WFDC5-Nand WFDC12-WAP domains share a common ancestral protein (Fig. 3).

Possible applications in therapeutics. Antimicrobial properties of recombinant human SLPI-N-WAP are observed not only in vivo against E. coli and Staphylococcus aureus, but also in vitro against the spread of fungi, bacteria, and viruses $(1,2)$. Since elderly individuals are particularly susceptible to mucosal infections and are known to produce diminished amounts of oral SLPI (2), these studies suggest that supplying recombinant WAPs to the saliva of elderly patients could prevent mucosal infections. Apparently, these recombinant proteins have advantages over many other protease inhibitors because they are not glycosylated and are much smaller $(\sim 5 \mathrm{kDa})$ than natural protease inhibitors such as serpins (40-50 kDa) (19) and cystatins (11-175 kDa) (20).

In summary, we have isolated 98 WAP homologues and based on their multiple alignments, we predict that most mammalian SLPI-N- and WFDC12-WAP domains have antimicrobial activities, whereas most mammalian SLPI-C- and elafin WAP domains have antiprotease activities. We also discussed the evolution of WAP family members. According to Fitch et al (1), increasingly virulent strains of bacteria and antibiotic resistance mean that proteinase inhibitors have the capacity to become the next 'magic bullet'. We are therefore continuing our efforts to collect WAP homologues, analyze their amino acid sequences, and predict in silico whether these homologues could have novel antimicrobial and/or antiprotease activities.

\section{Acknowledgements}

We thank Dr Etsuro Yamanishi, President of Hirakata Ryoikuen, for his constant support and encouragement. We thank the National Center for Biotechnology Informatics, USA; Genomics Institute of the Novartis Research Foundation, USA; DNA Data Bank of Japan; Computational Biology Research Center, AIST, Japan and the Ensembl, Wellcome Trust Sanger Institute, UK for access to the network servers.

\section{References}

1. Fitch PM, Roghanian A, Howie SEM and Sallenave JM: Human neutrophil elastase inhibitors in innate and adaptive immunity. Biochem Soc Trans 34: 279-282, 2006.

2. Shugars DC, Watkins CA and Cowen HJ: Salivary concentration of secretory leukocyte protease inhibitor, an antimicrobial protein, is decreased with advanced age. Gerontology 47: 246-253, 2001

3. Su AI, Cooke MP, Ching KA, et al: Large-scale analysis of the human and mouse transcriptomes. Proc Natl Acad Sci USA 99: 4465-4470, 2002.

4. Clauss A, Lilja $\mathrm{H}$ and Lundwall A: The evolution of a genetic locus encoding small serine proteinase inhibitors. Biochem Biophys Res Commun 333: 383-389, 2005.

5. Bono H, Yagi K, Kasukawa T, et al: Systemic expression profiling of the mouse transcriptome using RIKEN cDNA microarrays. Genome Res 13: 1318-1328, 2003.

6. Puente XS and Lopez-Otin C: A genomic analysis of rat proteases and protease inhibitors. Genome Res 14: 609-622, 2004.

7. Altschul SF, Madden TL, Schaffer AA, et al: Gapped BLAST and PSI-BLAST: a new generation of protein database search programs. Nucleic Acids Res 25: 3389-3402, 1997.

8. Marchler-Bauer A, Panchenko AR, Shoemaker BA, et al: CDD: a database of conserved domain alignments with links to domain three-dimensional structure. Nucleic Acids Res 30: 281$283,2002$.

9. Thompson JD, Higgins DG and Gibson TJ: CLUSTAL W: Improving the sensitivity of progressive multiple sequence alignment through sequence weighting, position-specific gap penalties and weight matrix choice. Nucleic Acids Res 22: 4673-4680, 1994.

10. Saitou $\mathrm{N}$ and Nei M: The neighbor-joint method: a new method of reconstructing phylogenetic tree. Mol Biol Evol 4: 406-425, 1987.

11. Tamura K, Dudley J, Nei M and Kumar S: MEGA4: Molecular evolutionary genetics analysis (MEGA) software version 4.0. Mol Biol Evol 24: 1596-1599, 2007.

12. Hurle B, Swanson W, NISC Comparative Sequencing Program, and Green ED: Comparative sequence analyses reveal rapid and divergent evolutionary changes of the WFDC locus in the primate lineage. Genome Res 17: 276-286, 2007.

13. Hagiwara K, Kikuchi T, Endo Y, et al: Mouse SWAM1 and SWAM2 are antibacterial proteins composed of a single whey acidic protein motif. J Immunol 170: 1973-1979, 2003.

14. Grutter MG, Fendrich G, Huber R and Bode W: The 2.5 A X-ray crystal structure of the acid-stable proteinase inhibitor from human mucous secretions analyzed in its complex with bovine alfa-chymotrypsin. EMBO J 7: 345-351, 1988. 
15. Tsunemi M, Matsuura Y, Sakakibara S and Katsube Y: Crystal structure of an elastase-specific inhibitor elafin complexed with porcine pancreatic elastase determined at 1.9 A resolution. Biochemistry 35: 11570-11576, 1996.

16. Brown TI, Mistry R, Collie DD, Tate S and Sallenave JM: Trappin ovine molecule (TOM), the ovine ortholog of elafin, is an acute phase reactant in the lung. Physiol Genomics 19: 11-21, 2004.

17. Wright CD, Kennedy JA, Zitnik RJ and Kashem MA: Inhibition of murine neutrophil serine proteinases by human and murine secretory leukocyte protease inhibitor. Biochem Biophys Res Commun 254: 614-617, 1999.
18. Brown TI, Mistry R, Gray R, Imrie M, Collie DD and Sallenave JM: Characterization of the ovine ortholog of secretory leukoprotease inhibitor. Mamm Genome 16: 621-630, 2005.

19. van Gent D, Sharp P, Morgan K and Kalsheker N: Serpins: structure, function and molecular evolution. Int J Biochem Cell Biol 35: 1536-1547, 2003.

20. Bobek LA and Levine MJ: Cystatins - inhibitors of cysteine proteinases. Crit Rev Oral Biol Med 3: 307-332, 1992. 